Research Paper

\title{
Dietary Flaxseed Mitigates Impaired Skeletal Muscle Regeneration: in Vivo, in Vitro and in Silico Studies
}

\author{
Felicia Carotenuto ${ }^{1,2^{*}}$, Alessandra Costa ${ }^{3,4}$, Maria Cristina Albertini ${ }^{5}$, Marco Bruno Luigi Rocchi ${ }^{5}$, Alexander \\ Rudov $^{5}$, Dario Coletti ${ }^{6}$, Marilena Minieri , Paolo Di Nardo ${ }^{1}$ and Laura Teodori2 ${ }^{\bowtie}$ \\ 1. Department of Clinical Sciences and Translational Medicine, University of Rome Tor Vergata, Rome, Italy. \\ 2. Diagnostic \& Metrology, FSN-TECFIS-DIM, ENEA, Frascati-Rome, Italy \\ 3. Department of Surgery, McGowan Institute, University of Pittsburgh Medical Center, Pittsburgh, PA, USA. \\ 4. Fondazione San Raffaele, Ceglie Messapica Italy. \\ 5. Department of Biomolecular Sciences; Urbino University "Carlo Bo"; Urbino, Italy. \\ 6. UMR 8256, UPMC P6, Pierre et Marie Curie University, Department of Biological Adaptation and Aging, Paris Cedex, France. \\ 7. Department of Experimental Medicine and Surgery, University of Rome Tor Vergata , Rome, Italy. \\ *Visiting Researcher at FSN-TECFIS-DIM, ENEA \\ $\triangle$ Corresponding author: Laura Teodori, Diagnostic \& Metrology, FSN-TECFIS-DIM, ENEA, via Enrico Fermi 45 I-00044 Frascati, Rome Italy; Phone: \\ +39-06-94005642; Email: laura.teodori@enea.it.
}

( ) Ivyspring International Publisher. Reproduction is permitted for personal, noncommercial use, provided that the article is in whole, unmodified, and properly cited. See http://ivyspring.com/terms for terms and conditions.

Received: 2015.07.17; Accepted: 2015.10.24; Published: 2016.02.18

\begin{abstract}
Background: Diets enriched with n-3 polyunsaturated fatty acids ( $n-3$ PUFAs) have been shown to exert a positive impact on muscle diseases. Flaxseed is one of the richest sources of $n-3$ PUFA acid $\alpha$-linolenic acid (ALA). The aim of this study was to assess the effects of flaxseed and ALA in models of skeletal muscle degeneration characterized by high levels of Tumor Necrosis Factor- $\alpha$ (TNF).

Methods: The in vivo studies were carried out on dystrophic hamsters affected by muscle damage associated with high TNF plasma levels and fed with a long-term 30\% flaxseed-supplemented diet. Differentiating $\mathrm{C} 2 \mathrm{C} 12$ myoblasts treated with TNF and challenged with ALA represented the in vitro model. Skeletal muscle morphology was scrutinized by applying the Principal Component Analysis statistical method. Apoptosis, inflammation and myogenesis were analyzed by immunofluorescence. Finally, an in silico analysis was carried out to predict the possible pathways underlying the effects of $n-3$ PUFAs.

Results: The flaxseed-enriched diet protected the dystrophic muscle from apoptosis and preserved muscle myogenesis by increasing the myogenin and alpha myosin heavy chain. Moreover, it restored the normal expression pattern of caveolin-3 thereby allowing protein retention at the sarcolemma. ALA reduced TNF-induced apoptosis in differentiating myoblasts and prevented the TNF-induced inhibition of myogenesis, as demonstrated by the increased expression of myogenin, myosin heavy chain and caveolin-3, while promoting myotube fusion. The in silico investigation revealed that FAK pathways may play a central role in the protective effects of ALA on myogenesis.

Conclusions: These findings indicate that flaxseed may exert potent beneficial effects by preserving skeletal muscle regeneration and homeostasis partly through an ALA-mediated action. Thus, dietary flaxseed and ALA may serve as a useful strategy for treating patients with muscle dystrophies.
\end{abstract}

Key words: muscle dystrophy; diet; flaxseed; Inflammation; myogenesis; in silico.

\section{Introduction}

Adult skeletal muscles represent a plastic organ endowed with a remarkable capacity to regenerate in response to injury [1]. The leading role in muscle growth and regeneration is played by satellite cells, which are anatomically positioned between the myo- fiber sarcolemma and basal lamina [2] in quiescent conditions, and are rapidly activated to form new myofibers in response to appropriate stimuli [3]. In normal skeletal muscle, regeneration is a coordinated process in which several factors are sequentially acti- 
vated to maintain and preserve muscle structure and function. However, in some disease states, such as muscular dystrophy, this regenerative capacity is impaired [4,5]. Dystrophies are hereditary skeletal muscle degenerative diseases caused by mutations in genes, most of which encode for proteins that are indispensable to the integrity of the muscle cell membrane and structure [6]. Their functional absence enhances muscle susceptibility to mechanical and biochemical injuries, with consequent membrane leakage and loss of muscle cells [7]. Dystrophic muscle is characterized by progressive myofiber loss, chronic local inflammation and fibrosis, which in severe forms lead to paralysis, respiratory and cardiac failure and, eventually, the death of the patient. Indeed, the chronic inflammatory response creates a hostile microenvironment that inhibits the regenerative capacity of the satellite stem cells responsible for exacerbating the deleterious processes [4]. Therefore, modulation of the inflammatory signals in the muscle microenvironment represents a critical topic for current investigations. Nutritional factors may target critical players involved in inflammation response, tissue regeneration and repair [8]. Previous studies have demonstrated that dietary flaxseed exerts an anti-inflammatory effect in animals [9] and humans [10] by reducing circulating inflammatory molecules. Flaxseed is one of the richest sources of n3-PUFA acid $\alpha$-linolenic acid (ALA) [11]. Our previous studies have demonstrated that dietary supplementation with flaxseed prevents the fibrosis and derangement of skeletal and cardiac muscle structure and function in dystrophic hamsters, markedly extending the animals' lifespan [12-14]. In this animal model, characterized by a deletion of the $\delta$-sarcoglycan gene, muscle damage occurs early and peaks when the animal is 120-150 days old. This late phase of disease is characterized by increased plasma levels of tumor necrosis factor- $\alpha$ (TNF) [13]. TNF is a major pro-inflammatory cytokine that is expressed in damaged skeletal muscle; increased TNF levels have been found in the plasma and muscles of dystrophic animals and humans [15]. High TNF plasma levels $(0.5-10 \mathrm{ng} / \mathrm{ml})$ have been associated with myoblast and myocyte apoptosis, inhibition of myogenic differentiation and muscle wasting [16-19]. Dietary flaxseed has been shown to counteract the deleterious effects of TNF on cardiac muscle cell survival through a mechanism that regulates caveolin-3 expression and accumulation in caveolae and is likely due to the ALA content of flaxseed [13]. Caveolin-3 is a muscle-specific protein involved in cardiac and skeletal muscle protection [20-22] and is essential for myoblast fusion and myotube formation [23]. These findings suggest that flaxseed and its n3 fatty acid ALA may be able to coun- teract the harmful effects of TNF on myogenesis. Therefore, in the present study, we decided to investigate the possible beneficial effects of flaxseed and ALA in models of TNF-induced impaired myogenic differentiation. The in vivo model we adopted was the dystrophic hamster (Dystr/P), characterized by increased TNF plasma levels associated with skeletal muscle degeneration, which was fed with a flaxseed-enriched diet (FS diet) from weaning to death. Murine myoblasts treated with high concentrations of TNF and challenged with ALA represented the in vitro model. In addition, to identify the mechanisms and pathways underlying the effects of flaxseed and ALA on skeletal muscle, we carried out an in silico analysis. In view of the findings of previous studies that highlighted a possible epigenetic mechanism through which n-3 PUFAs regulate protein expression [24-26], we also performed an extensive literature search to identify putative microRNAs (miRNA) likely to underlie the effects of n-3 PUFAs on impaired myogenesis.

MicroRNAs (miRNAs) are 18-25 nucleotide non-coding RNAs that post-transcriptionally regulate gene expression by stalling the translation of the cognate mRNA or by promoting its degradation [27]. MicroRNAs are emerging as prominent players in myogenic differentiation [28] and represent a level of molecular regulation through which hundreds of genes involved in different signaling pathways can be regulated simultaneously [29]. Therefore, miRNAs can be used as a tool to uncover the pathways and targets that underlie changes in skeletal muscle in different pathophysiological conditions and after specific treatments. To identify the miRNA targets and pathways that are shared by more than one miRNA, a computer program named SID1.0 (simple String IDentifier) was developed [30]. This string identification program has proven to be a very useful tool to predict new genes, miRNAs and related targets and pathways involved in different pathophysiological processes $[30,31]$. In the present study, we performed the in silico analysis of the pathways shared by different miRNAs involved in the effects of $n-3$ PUFAs on myogenesis to support the experimental in vivo and in vitro observations.

\section{Materials and methods}

\section{In Vivo}

\section{Animals and Dietary Treatment}

Syrian hamsters (strain UM-X7.1), in which a deletion of the $\delta$-sarcoglycan gene $(\delta$-SG) determines a hereditary dystrophy that reproduces the human LGMD2F [32] phenotype, were used in the present study. Dystrophic hamsters were randomly divided 
in 2 groups: the first group (Dystr/P group) was fed with standard pellet chow (Rieper SpA), the second group (Dystr/FS group) with a 30\% flaxseed-supplemented diet (FS diet). Golden Syrian hamsters bred under the same conditions and fed with standard pellet chow $(\mathrm{P})$ were used as healthy controls (Healthy group). All animals were allowed to consume food ad libitum from weaning to sacrifice. The FS diet consisted of whole brown flaxseed, apples and carrots (30:50:20 w/w), with flaxseed (FS) being the only source of fats. The diet composition analysis, which was previously reported [14], showed that all macro- and micro-nutrients were quantitatively adequate to maintain the animals healthy in both dietary regimens. This flaxseed diet has been recognized as source of n-3 PUFAs, with ALA representing $52 \%$ of the total lipids $[11,33]$ and is referred to throughout the paper as the FS diet. The average daily amount of flaxseed eaten by each animal was $2.1 \mathrm{~g} /$ day/100g body weight. The caloric power in $100 \mathrm{~g}$ of fresh Pellet or FS diet was $222.5 \pm 48$ and $202.8 \pm 45 \mathrm{kcal}$, respectively. Every 7 days, animal weights were recorded to exclude possible decreases attributable to calorie restriction. All the observations were made on 150-day-old animals, i.e. an age when muscular dysfunction and degeneration is severe and clearly evident.

\section{Hamster Tissue Sampling}

The study protocol was preliminarily approved by the Animal Care Committee of the Tor Vergata University of Rome (Italy) and performed in accordance with the Directive 2010/63/EU of the European Parliament. Hamsters were anesthetized with urethane $(400 \mathrm{mg} / \mathrm{kg}$ ip) and sacrificed at 150 days of age. Blood was collected by ventricular puncture, centrifuged and the plasma was stored at $-80^{\circ} \mathrm{C}$ until use. Biceps femoris muscles were rapidly excised, washed in cold PBS, frozen in liquid nitrogen and stored at $-80^{\circ} \mathrm{C}$ until use. Alternatively, muscles were fixed with $4 \%$ paraformaldehyde and embedded in paraffin for microscopy analysis. At least 5 animals per group were considered for each analysis.

\section{Histological analysis}

Histological sections $(4-\mu \mathrm{M})$ were cut from paraffin-embedded skeletal muscles, deparaffinized in xylene, rehydrated in ethanol and stained with H\&E (Bio-Optica, Milan, Italy) according to standard procedures in order to quantify the morphological observation. The images were acquired by means of a Leica DMRB microscope coupled with a digital camera.

To determine the percentage of myofibers with internalized nuclei, micrographs of H\&E stained skeletal muscle sections were captured using a digital camera, and fibers with internalized nuclei were counted using NIH ImageJ software from five sections taken from each hamster ( $\mathrm{n}=5$ animals/group).

\section{Principal Component Analysis on histological section}

The images of H\&E stained skeletal muscle sections (from six sections taken from each hamster, $n=5$ animals/group) were processed by ImageJ software (http://imagej.nih.gov/ij/docs/intro.html). All the commands related to statistical measurements on image data, profile and histogram plotting and plugins related to image analysis can be found in http://imagej.nih.gov/ij/docs/guide/146-30.html\#t oc-Subsection-30.7. The following morphometric parameters were selected as being the most representative of fiber shape in the skeletal muscle: Area (area of selection in square pixels); Circ: (calculate to display circularity), AR (aspect ratio, major axis/minor axis); Round: (roundness); Solidity (area/convex area). Extracellular Area represents the extension of extracellular matrix. Circ, AR and Round represent the shape descriptors.

To detect any correlations between groups characterized by different morphologies, a principal components analysis (PCA) on the aforementioned morphometric parameters was carried out. The PCA is a procedure for analyzing multivariate data designed to reduce the dimensionality of the data and allow the visualization of a large number of variables on a two-dimensional plot [34-36].

Three groups were considered in the in vivo experiments, i.e. Healthy, Dystr/P and Dystr/FS, as well as six variables that correspond to the morphological parameters described above. A diagram of the values obtained from images for each group was plotted in bidimensional space, defined by the $1^{\text {st }}$ and $2^{\text {nd }}$ Principal component functions on the $x$-axis and $\mathrm{y}$-axis, respectively (PC1 and $\mathrm{PC} 2$ ). The variables correlated with the two principal components can be identified by the highest score coefficients in absolute values. A multivariate analysis of variance (ANOVA) was performed to compare the groups with regard to the variables extracted from the image analysis. Moreover, a univariate ANOVA test was applied for each variable, followed by a multiple comparison between the three groups performed by means of the least significance difference (LSD) test. The significance level was set at alpha $=0.05$. Statistical analyses were performed with SPSS 18.0. (Statistical Package for Social Sciences). For each PCA the variance explained by the model was evaluated [37].

\section{Tumor necrosis factor-alpha plasma levels}

Hamsters TNF plasma levels were measured 
using a specific enzyme-linked immunosorbent assay (ELISA) (R\&D Inc.), according to the manufacturer's instructions. Values were from 6 animals/group.

\section{Apoptosis quantification}

TUNEL assay was performed on paraffin hamster muscular sections using the terminal deoxynucleotidyl transferase (TdT)-mediated in situ fluorescein-conjugated, dUTP nick end-labeling technique (In Situ Cell Death Detection Kit, Fluorescein), according to the manufacturer's protocol (Roche Diagnostic Corp.). Briefly, muscle sections (biceps femoris) were deparaffinized in xylene, rehydrated and then treated with proteinase-K before proceeding with the assay. Sections were stained with a mouse monoclonal anti-a-sarcomeric-actin (Sigma-Aldrich) and then with anti-mouse Alexa 546 secondary antibodies (Molecular Probes). Nuclei were stained with DAPI. To determine the percentage of apoptotic cells, micrographs of skeletal muscle sections were captured using a Leica microscope (Leica Microsystems DMRB), and positive and negative TUNEL nuclei were counted using NIH ImageJ software from five sections taken from each hamster ( $\mathrm{n}=5-6$ animals/group).

\section{Immunofluorescence}

Paraffin-embedded muscle sections were deparaffinized in xylene, rehydrated, and then processed in $10 \mathrm{mM}$ citric acid ( $\mathrm{pH}$ 6.0) by microwave for antigen retrieval treatment. The sections were then incubated in $8 \%$ BSA in PBS and stained with anti CD45, monoclonal (Santa Cruz), anti-alpha sarcomeric Actin, Polyclonal, (Thermo Scientific Pierce), rabbit anti-Pax 3-7, goat Mab ( Santa Cruz Biotechnology); anti-myogenin, mouse Mab (BD Transduction), anti- $\alpha-M H C$, mouse Mab (from Stefano Schiaffino), rabbit polyclonal anti caveolin-3 (Abcam) followed by an appropriate secondary antibody: anti-mouse 546 Alexa Fluor, anti-rabbit 546 Alexa Fluor, anti-rabbit 488 Alexa Fluor and anti-goat 488 Alexa Fluor, (Molecular Probes). To label the sarcolemma, TRITC-Wheat germ agglutinin (TRITC-WGA, Sigma-Aldrich) was dissolved in PBS and applied at a final concentration of $20 \mu \mathrm{g} / \mathrm{ml}$ for $1 \mathrm{~h}$ at room temperature, after slide incubation with the secondary antibody. After nucleus staining with DAPI (Sigma-Aldrich), skeletal muscle sections were analyzed by means of a fluorescence microscope (Leica Microsystems DMRB). All quantifications were performed using ImageJ software (http://rsb.info.nih.gov/ij/). To determine the percentage of positive CD45 cells and positive Pax7 and myogenin nuclei, micrographs of skeletal muscle sections were captured and cells counted from six sec- tions taken from each hamster, $\mathrm{n}=5$ animals/group. To determine the fluorescence of $\alpha-\mathrm{MHC}$ and caveolin-3 levels in skeletal muscle sections, the total cell fluorescence (CTCF) was calculated from digital images using the following formula [38]:

\section{$\mathrm{CTCF}=$ Integrated Density $-($ Area of selected cell X \\ Mean fluorescence of background readings)}

Images were from six sections, taken from each hamster ( $\mathrm{n}=6$ animals/group).

\section{Northern Blot}

Total RNA was extracted from frozen skeletal muscle samples using TRIZol Reagent (Sigma-Aldrich), according to the manufacturer's specifications, and an aliquot $(20 \mu \mathrm{g})$ was electrophoresed on a $1.25 \%$ agarose gel containing $5 \%$ formaldehyde and transferred to Hybond $\mathrm{N}$ membrane (Amersham Corp., Arlington Heights, IL). Northern blot analysis of $\alpha$-sarcomeric actin and myomesin was performed as previously described [14]; cDNA probes were kindly provided by Jean-Claude Perriard and Fabrizio Loreni. The Scion Image software was used to quantify band intensity.

\section{In vitro}

\section{Cell Cultures}

Murine C2C12 myoblasts (American Type Culture Collection) were cultured in growth media (GM) consisting of Dulbecco's modified Eagle's medium (DMEM) supplemented with 15\% Fetal Bovine Serum (FBS) and $50 \mathrm{mg} / \mathrm{ml}$ gentamicin (Sigma-Aldrich, St. Louis, MO) and seeded at a density of $10^{4}$ cell $/ \mathrm{cm}^{2}$ onto multiwell plates or flasks. To induce differentiation, when at $70 \%$ confluence, cells were shifted to a differentiating medium (DM) consisting of DMEM supplemented with $2 \%$ fetal horse serum and 50 $\mathrm{mg} / \mathrm{ml}$ gentamicin for 2 or 5 days. Cell cultures were treated with $10 \mathrm{ng} / \mathrm{ml}$ mouse recombinant TNF (Sigma-Aldrich) added to DM in order to mimic the in vivo inflammation environment for 2 or 5 days in the presence or absence of ALA. ALA was preliminarily complexed with fatty acid free bovine serum albumin (BSA fraction V, Sigma-Aldrich, fatty acid/BSA molar ratio 4:1) and added to DM at a concentration of 10 $\mu \mathrm{M}$.

\section{Principal Component Analysis on cell culture}

The morphometric parameters derived from light microscope images of H\&E stained C2C12 cells were elaborated with the image processing software (ImageJ), as previously described for in vivo studies. The following morphometric parameters were considered: Area; StdDev (standard deviation of the gray values used to generate the mean gray value); $\mathbf{X}$ and $\mathbf{Y}$ 
Centroid (the average of the $\mathrm{x}$ and $\mathrm{y}$ coordinates of all of the pixels in the image or selection which uses the $X$ and $\mathbf{Y}$ headings); $\mathbf{X M}$ and $\mathbf{Y M}$ Center of Mass (the brightness-weighted average of the $\mathrm{x}$ and $\mathrm{y}$ coordinates all pixels in the image or selection which uses the $\mathrm{XM}$ and $\mathrm{YM}$ headings); Perimeter (perimeter, the length of the outside boundary of the selection); the shape descriptors: AR; Round; Solidity; Circ; Num Nuclei (the number of nuclei in each cell). The relationship between groups characterized by different morphologies was assessed by PCA. In in vitro experiments, four groups were considered: CTR/GM, CTR/DM, TNF and TNF ALA, together with the twelve variables that correspond to the morphological parameters described above. A diagram of the values obtained from images for each group was plotted in the bidimensional space, defined by the $1^{\text {st }}$ and $2^{\text {nd }}$ Principal components on the $\mathrm{x}$-axis and $\mathrm{y}$-axis, respectively (PC1 and PC2). The variables correlated with the two principal components can be identified by the highest score coefficients in absolute values. A multivariate analysis of variance (ANOVA) was performed to compare the groups with regard to the variables extracted from the image analysis. Moreover, a univariate ANOVA test was applied for each variable, followed by a multiple comparison between the three groups performed by using the least significance difference (LSD) test. The significance level was set at alpha $=0.05$. Statistical analyses were performed with SPSS 18.0. (Statistical Package for Social Sciences). The variance explained by the model was evaluated for each PCA.

\section{Apoptosis quantification}

To analyze apoptotic nuclei by TUNEL assay, C2C12 cells were fixed in $4 \%$ paraformaldehyde in PBS, pH 7.4, and permeabilized with $0.1 \%$ Triton $\mathrm{X}-100$ in $0.1 \% \mathrm{Na}$ citrate. TUNEL assay was performed using the terminal deoxynucleotidyl transferase (TdT)-mediated in situ fluorescein-conjugated, dUTP nick end-labeling technique (In Situ Cell Death Detection Kit, Fluorescein), according to the manufacturer's protocol (Roche Diagnostic Corp.). To assess cell apoptosis, nuclei were analyzed from 10 random fields selected from 5 cell wells for each treatment using NIH ImageJ software. Apoptotic cell counts were expressed as the percentage of the total number of nuclei counted.

\section{Immunofluorescence}

Primary antibodies used for immunofluorescence were: monoclonal anti-myogenin mouse Mab (BD Transduction), MF20 monoclonal antibody (MAb) (Developmental Studies Hybridoma Bank, University of Iowa) and mouse anti-caveolin-3 (BD
Transduction Laboratories $\left.{ }^{\mathrm{TM}}\right)$. Cells were fixed with $4 \%$ paraformaldehyde and permeabilized with $0.1 \%$ Triton X-100 for 2 min and then incubated with primary antibody for $1 \mathrm{~h}$ and subsequently with an appropriate secondary antibody (488 Alexa Fluor, Molecular Probes) for $1 \mathrm{~h}$. After nucleus staining with 1 $\mu \mathrm{g} / \mathrm{ml}$ DAPI (Sigma-Aldrich), cells were analyzed using a fluorescence microscope (Leica Microsystems, Mod. DMRB) equipped with a digital camera. All quantifications were performed using ImageJ software. (http://rsb.info.nih.gov/ij/). To determine the percentage of positive myogenin nuclei above the total number of nuclei, and MHC and caveolin-3 positive cells above the total number of cells, micrographs were captured and cells counted from a minimum of six random fields selected from three slides.

The fusion index of $\mathrm{C} 2 \mathrm{C} 12$ myoblasts after specific treatment was calculated as the average number of nuclei in MHC positive cells containing at least three nuclei above the total number of nuclei.

\section{Statistical analysis}

Results are expressed as the mean \pm SD. The analysis of variance was performed (ANOVA) for comparisons between more than two groups, whereas the two-tailed unpaired Student's $t$-test was used for comparisons of the mean differences between two groups. A suitable post-hoc test was used in combination with ANOVA to test for significant differences between groups. Differences were considered statistically significant when $P<0.05$. (SPSS for Windows, version 11.5; SPSS, Inc., Chicago, IL).

\section{In silico}

A literature search was performed using PubMed or ISI Web of knowledge Databases to identify miRNAs with experimental evidence of involvement in murine muscle differentiation and modulated by n-3 PUFAs or TNF (last accessed December 21 2014). The following key words were used: miRNAs and differentiation and $\mathrm{C} 2 \mathrm{C} 12$ or TNF and miRNAs or $\mathrm{n}-3$ PUFAs and miRNAs. Because a lack of data was found, regarding the influence of ALA on microRNA we extended PubMed searches to n-3 PUFAs. Moreover, we extended PubMed searches to humans miRNAs, because, (at the best of our knowledge) no literature on n-3 PUFAs modulation of miRNAs in murine cells was found as yet. Thus, the murine miRNAs, which are equivalent to humans $\mathrm{mi}^{-}$ croRNAs, were considered in the present research. To obtain the common pathways of specific miRNAs, the list IDs of KEGGs pathway and database DIANA-mirPath [39] were indexed using SID1.0. Since a visual inspection of the IDs would be unpractical due to their large number (thousands IDs), they 
have been automatically indexed using a simple Fortran written program (SID1.0; String IDentifier), developed by us [30], that looks for Refseq IDs shared by the predicted pathways of the different datasets. The database also allows to report the $-\ln (p$-value) (the negative natural logarithm of the enrichment p-value calculated for the specific pathway). The p-value is a measure of the association between a selected gene from the list of pathways. In pathway analysis, generally p-values less than 0.05 indicate that the association is not statistically significant and the pathway could be rejected.

\section{Results}

\section{In Vivo}

Flaxseed diet improves the skeletal muscle architecture in dystrophic hamsters

Figure 1A shows haematoxylin/eosin staining of tissue sections from the hamster biceps femoris muscles. The normal muscles display regular polygonal fibers, a homogeneous diameter and peripheral nuclei located directly below the sarcolemma. The Dystr/P muscle sections revealed fibers that were rounded in shape and of different sizes. In addition, an increased number of fibers with internalized nuclei were observed, as quantified in Figure 1 B. Fibers under degeneration were also observed. The FS diet resulted in an increased myofiber size, a reduced variability of myofiber size, and markedly decreased numbers of fibers with internalized nuclei and of degenerated fibers (Fig. 1 A, B).

To quantify the effects of the FS diet on the morphology of the H\&E staining skeletal muscle sections, the PCA [34] was performed. Six morphometric parameters were opportunely selected by ImageJ software, as reported in the Materials and Methods section (Fig. 1C). The measurements of these parameters were used as variables to construct two Principal component functions. The diagram of the values (Fig.1D) obtained from the morphometric parameters for each group (Healthy, Dystr/P and Dystr/FS) showed that the Principal component 1 (PC1) was strongly correlated with the Area, AR and solidity parameters; the Principal component 2 (PC2) was strongly correlated with the Circ, Round and Extracellular Area parameters identified by the highest score coefficients in absolute values (Fig. 1C in the red box). Dystr/P (yellow dots) revealed a growing PC2 (y-axis value) compared with the Healthy group (blue dots) (the total variance explained by the PC1 and PC2 was $53.3 \%$ ) due to the round-shaped fibers and larger extracellular space (Fig. 1D). Despite being more dispersed than the other groups, the Dystr/FS group (green dots) clearly falls between the Dystr/P and
Healthy groups. It is noteworthy that the morphological parameters of the muscles of hamsters fed with flaxseed (Dystr/FS) restored the Circ, Round and Extracellular Area parameters (PC2), thereby bringing these values closer to those of healthy animals and demonstrating the occurrence of larger myofibers (Fig. 1D). These results support the conclusion that the FS diet can significantly attenuate the histopathological features of dystrophy in hamster models of the disease.

\section{Flaxseed diet reduces inflammation and cell death}

In order to determine whether the altered morphology in dystrophic skeletal muscles was associated with an inflammatory status, the level of plasma TNF in 150-days-old hamsters was investigated by ELISA analysis. TNF levels were markedly increased in plasma of Dystr/P (3.2 fold) vs. healthy controls (Fig. $2 \mathrm{~A})$. The FS diet induced a slight, non-significant decrease in the cytokine levels. Inflammation of hamster muscle tissue was also monitored by pan-leukocyte marker CD45 immunofluorescence, which demonstrated a marked increase in the number of inflammatory cells in the Dystr/P compared with the healthy muscle sections (Fig. 2B). By contrast, in the skeletal muscles of Dystr/FS, the number of CD45 positive cells significantly decreased. Quantitative data are shown in Figure 2C. The high level of inflammation was associated with a higher number of apoptotic cells in the skeletal muscle of dystrophic hamsters than in those of healthy controls, as detected by TUNEL assay. The majority of the TUNEL-positive cells were located between the sarcolemma and the basal lamina (Fig. 2D). The FS diet significantly reduced the percentage of apoptotic cells in the Dystr/P to a level similar to that of healthy controls. The reduction in the number of inflammatory and apoptotic cells was associated with a general improvement in tissue texture, as demonstrated by co-staining with alpha-sarcomeric actin (Fig. 2D). The quantitative analysis of the TUNEL assay is shown in Figure 2E.

\section{Flaxseed diet improves myogenesis and differentiation}

In pellet-fed dystrophic vs. healthy hamster muscles, the aforementioned apoptosis was associated with a high expression of Pax7, a marker of satellite cells, as detected by immunofluorescence. Cells expressing Pax7, located between the sarcolemma and the basal lamina, were markedly reduced in flaxseed-fed when compared with pellet-fed dystrophic hamsters (Fig. 3A). The quantification of the results is shown in Figure 3B. Conversely, myogenin-positive nuclei were increased in Dystr/FS when compared with Dystr/P muscles. (Fig. 3C). Quantitative data are shown in Figure 3D. 
A

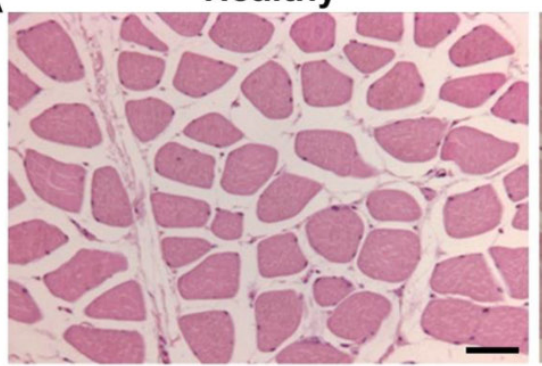

Dystr/P

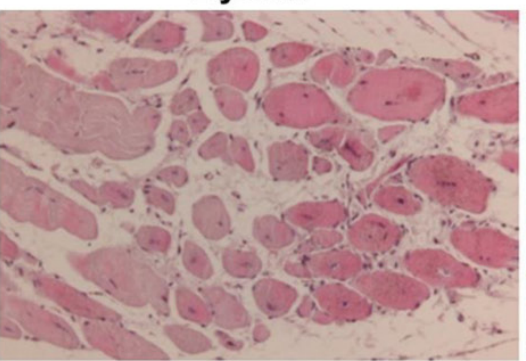

Dystr/FS

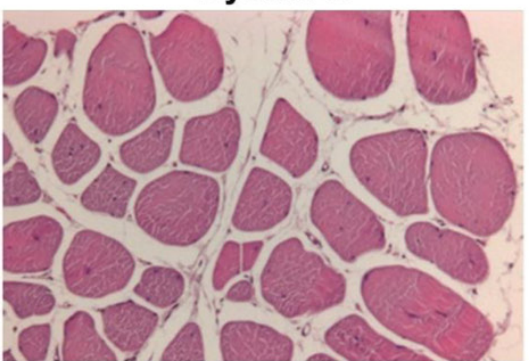

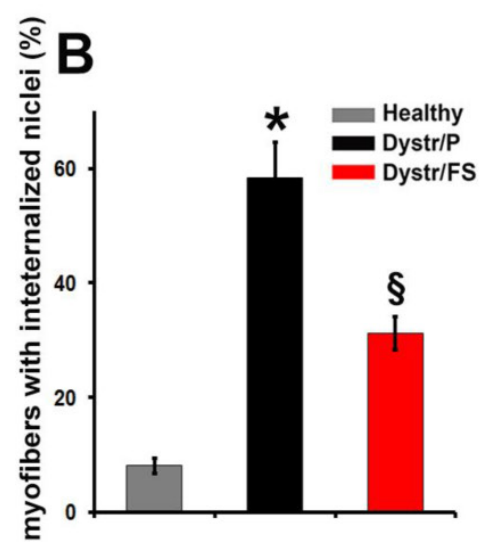

C

Variables Principal Component 1 Principal Component 2

Are

Circ

AR

Round

Solidity

Extracellular Area

0,182

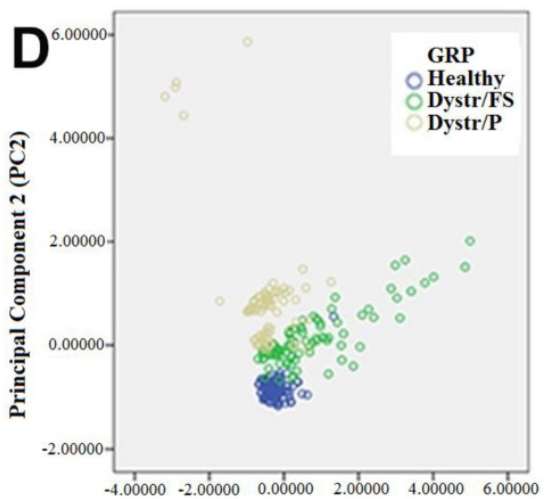

Principal Component 1 (PC1)

Figure 1. Flaxseed-enriched diet preserves dystrophic skeletal muscle morphology. For all in vivo observations, dystrophic hamsters were fed with a flaxseed-enriched diet (FS) from weaning to the age of 150 days (Dystr/FS) and compared with dystrophic (Dystr/P) and healthy hamsters (Healthy) fed with standard pellet (P). (A) Representative images of H\&E-stained skeletal muscle sections. Scale bar: $50 \mu \mathrm{m}$. (B) Percentage of myofibers with internalized nuclei from H\&E-stained skeletal muscle sections. ${ }^{*} P<0.05$ vs. Healthy; $\S P<0.05$ vs. Dystr/P; $n=5$. (C) Component Score Coefficient Matrix. The coefficients by which variables are multiplied to obtain factor scores are shown. The variables are represented by the morphometric parameters derived from light microscope images of skeletal muscle (six sections from each of 5 animals/group). The values highlighted (red boxes) indicate the variables most closely associated with Principal Components 1 and 2. (D) Principal Component Analysis (PCA). Three series of data from of Healthy, Dystr/FS and Dystr/P hamsters were plotted in the bidimensional space defined by the 1st and 2nd PCA. FS diet (Dystr/FS, green dots) restored the morphological parameters of the dystrophic (Dystr/P, yellow dots) phenotype towards value closer to those of healthy (blue dots) skeletal muscles.
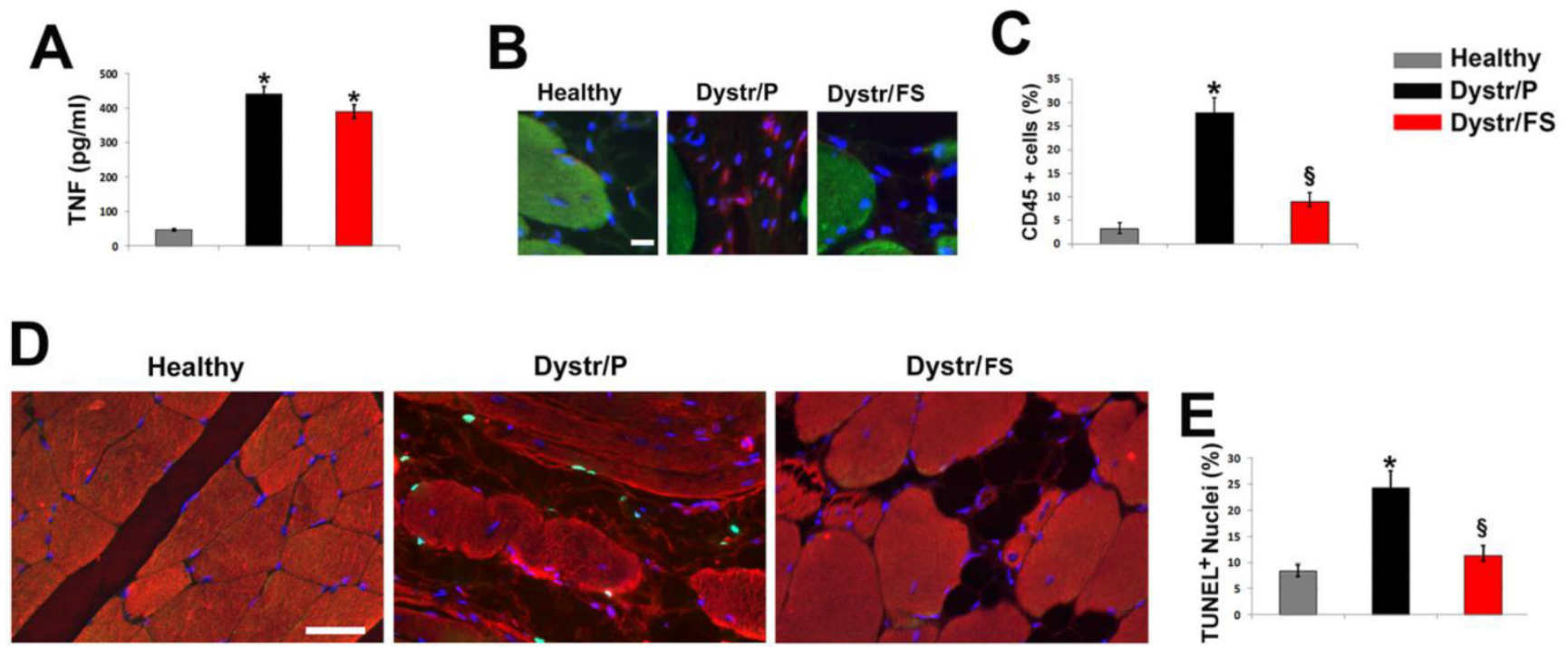

Figure 2. Flaxseed diet precludes inflammation and apoptosis in dystrophic skeletal muscle. (A) Hamster TNF plasma levels. $* \mathrm{P}<0.05$ vs. Healthy, $\mathrm{n}$ (number of animals per group) $=6$. (B) CD45 immunofluorescence (red) of skeletal muscle sections hamsters. $\alpha$-sarcomeric actin staining (green) highlights the skeletal fibers; nuclei were stained with DAPI (blue); Scale bar: $10 \mu \mathrm{m}$. (C) Percentage of CD45 positive cells vs. total number of cells in the hamster skeletal muscles. *P<0.05 vs. Healthy; $\S P<0.001$ vs. Dystr; $n=5$. (D) TUNEL assay of hamster skeletal muscle sections. Number of apoptotic nuclei (green) is higher in Dystr/P than in Healthy and Dystr/FS skeletal muscles. Muscle fibers are identified by $\alpha$-sarcomeric actin expression (red); nuclei were stained with DAPI (blue). Scale bar: $30 \mu \mathrm{m}$. (E) Quantitative analysis of TUNEL-positive nuclei as a percentage of total DAPI-stained nuclei. $* P<0.05$ vs. Healthy; $§ P<0.05$ vs. Dystr; $n=5-6$. 

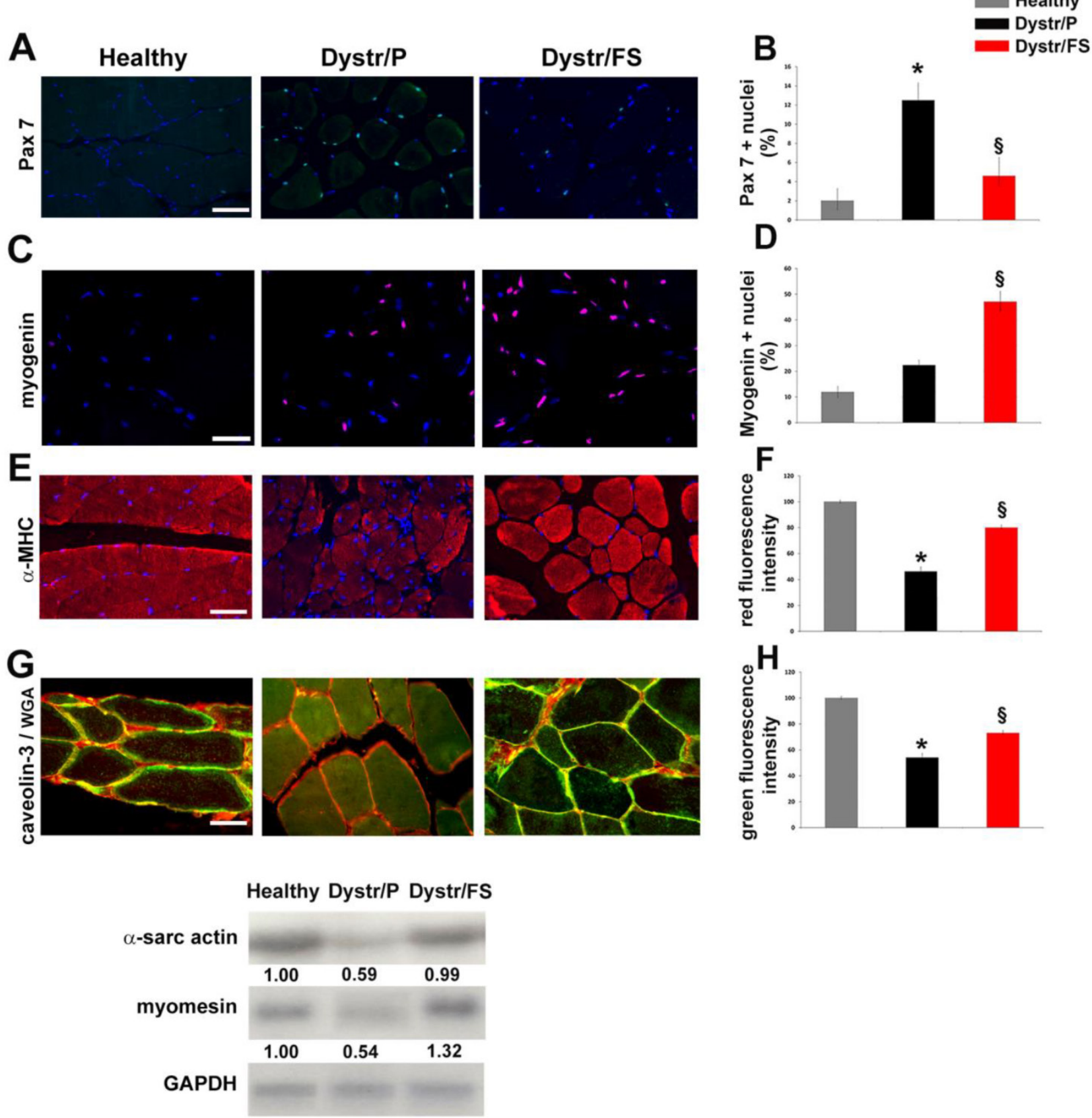

Figure 3. Flaxseed-enriched diet discontinues the negative degeneration/regeneration cycle in dystrophic skeletal muscle. (A) Pax7 immunofluorescence (green) of hamster skeletal muscle sections. Scale bar: $50 \mu \mathrm{m}$. Nuclei were stained with DAPI (Blue). (B) Bars represent the percentage of Pax7 positive cells of total DAPI-stained nuclei in hamster skeletal muscle sections. $* P<0.05$ vs. Healthy; $\S P<0.001$ vs. Dystr/P; $n=5$. (C) Myogenin staining (red) in hamster skeletal muscle sections. Nuclei were stained with DAPI (Blue). Scale bar: $50 \mu \mathrm{m}$. (D) Histogram showing the percentage of myogenin-positive nuclei out of all DAPI-stained nuclei in hamster skeletal muscle sections. $\S P<0.05$ vs. Dystr/P; $n=5$. (E) $\alpha-M H C$ immunofluorescence (red) of hamster skeletal muscle sections. Scale bar: $50 \mu$ m. (F) Quantification of $\alpha-M H C$ immunofluorescence (fiber/Area). Bars represent the average of total fluorescence intensity from each digital image calculated by ImageJ software. The values are expressed as a percentage of the healthy. $* P<0.05$ vs. Healthy; $\$ P<0.05$ vs. Dystr/P; $n=6$. (G) Representative images of caveolin-3 (green) and WGA-TRITC (sarcolemma marker, red) co-staining of hamster skeletal muscle sections. Merged images show that caveolin-3 expression increased in Dystr/FS vs. Dystr/P. The protein was mainly located in sarcolemma (yellow) in Healthy and Dystr/FS hamsters and in the cytosol in the Dystr/P animals, Scale bar: $20 \mu m$. (H) Quantification of caveolin-3 immunofluorescence (fiber/Area). Bars represent the average of the total green fluorescence intensity from each digital image calculated by ImageJ software. The values are expressed as a percentage of the healthy. ${ }^{*} P<0.05$ vs. Healthy; $\S P<0.001$ vs. Dystr/P; $n=6$. (I) The mRNA expression of $\alpha$-sarcomeric actin and M-band marker myomesin in hamster skeletal muscle samples. Gapdh was used as an internal control. Band intensities are expressed as fold increases compared to those of healthy animals.

To better understand the effects of the FS diet on muscle regenerative processes, we also tested the markers of advanced muscle differentiation, such as $\alpha-\mathrm{MHC}$ and caveolin-3. Figures $3 \mathrm{E}$ and $\mathrm{G}$ show that
$\alpha-\mathrm{MHC}$ and caveolin-3 levels were up-regulated by the administration of flaxseed to dystrophic animals. The quantification of the expression of the $\alpha-M H C$ and caveolin-3 signals are reported in panels $\mathrm{F}$ and $\mathrm{H}$ 
of Figure 3, respectively. In addition, caveolin-3 (co-localized with WGA) was decreased in the sarcolemma of Dystr/P compared with healthy muscles. Flaxseed administration induced the correct caveolin-3 localization within the sarcolemma in a fashion that was comparable to that of healthy hamster muscles (Fig. 3G). In addition, Northern blot analysis revealed higher amounts of $\alpha$-sarcomeric actin and M-band marker myomesin, which points to the formation of sarcomeres, in Dystr/FS than in Dystr/P muscles (Fig. 3I).

\section{In Vitro}

ALA preserves the morphology of differentiating myoblast $\mathrm{C} 2 \mathrm{C} 12$ by attenuating the deleterious effects of TNF

To test the effects of ALA on the inhibition induced by TNF on myogenesis, murine $\mathrm{C} 2 \mathrm{C} 12$ myoblasts were used as an in vitro cell model to recapitulate myogenic differentiation. After 5 days in DM,
TNF inhibited differentiation and myotube formation in $\mathrm{C} 2 \mathrm{C} 12$ cells, as shown in the H\&E stained image (Fig. 4A), thus confirming previous observations [40]. The addition of ALA to the DM prevented the inhibitory effect of TNF on myogenesis, thereby allowing the formation of myotubes in $\mathrm{C} 2 \mathrm{C} 12$ cells. (Fig. $4 \mathrm{~A}$ ).

To quantify any morphological changes, the PCA analysis was elaborated from twelve morphometric parameters opportunely selected and measured by ImageJ software. The resulting diagram (Fig. 4C) demonstrated that the Principal component 1 (PC1) was strongly associated with the Perim, AR, Circ, Num Nuclei, Area, Round and Solidity parameters, while the Principal component 2 (PC2) was strongly associated with StdDev, X, XM, Y and YM (red square in Fig. 4B). The PC1 varied markedly in differentiating (CTR/DM, green dots) and ALA-treated cells (TNF ALA, purple dots) when compared with the other groups (Fig. 4C).

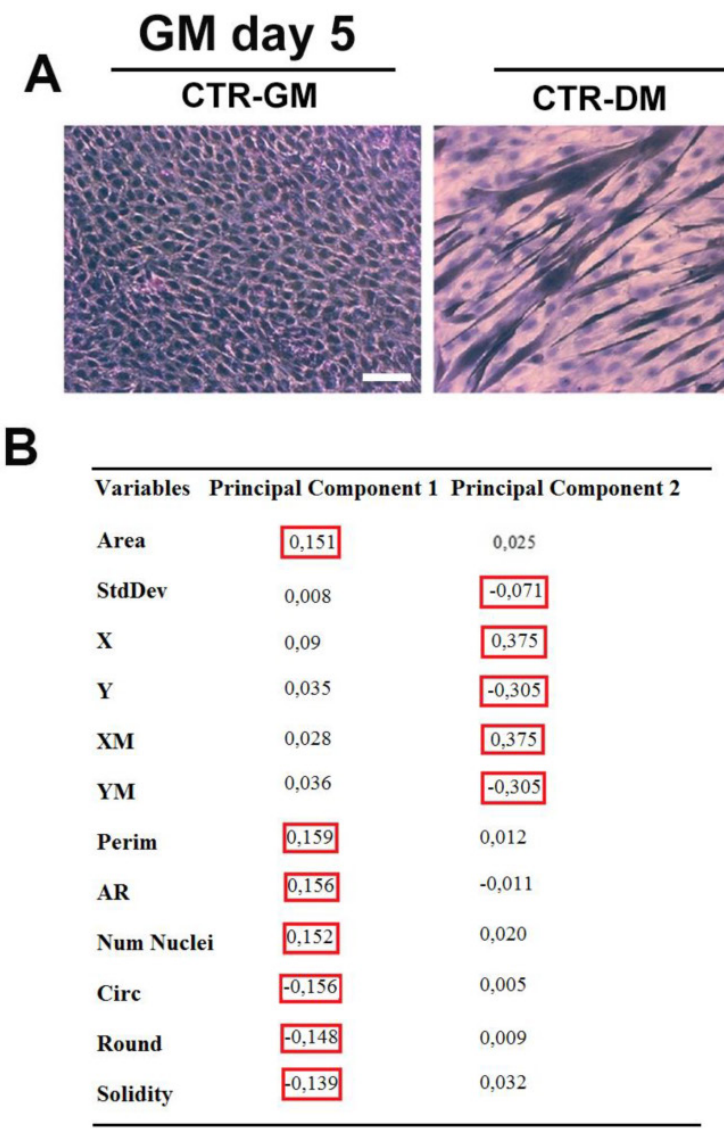

\section{DM day 5}
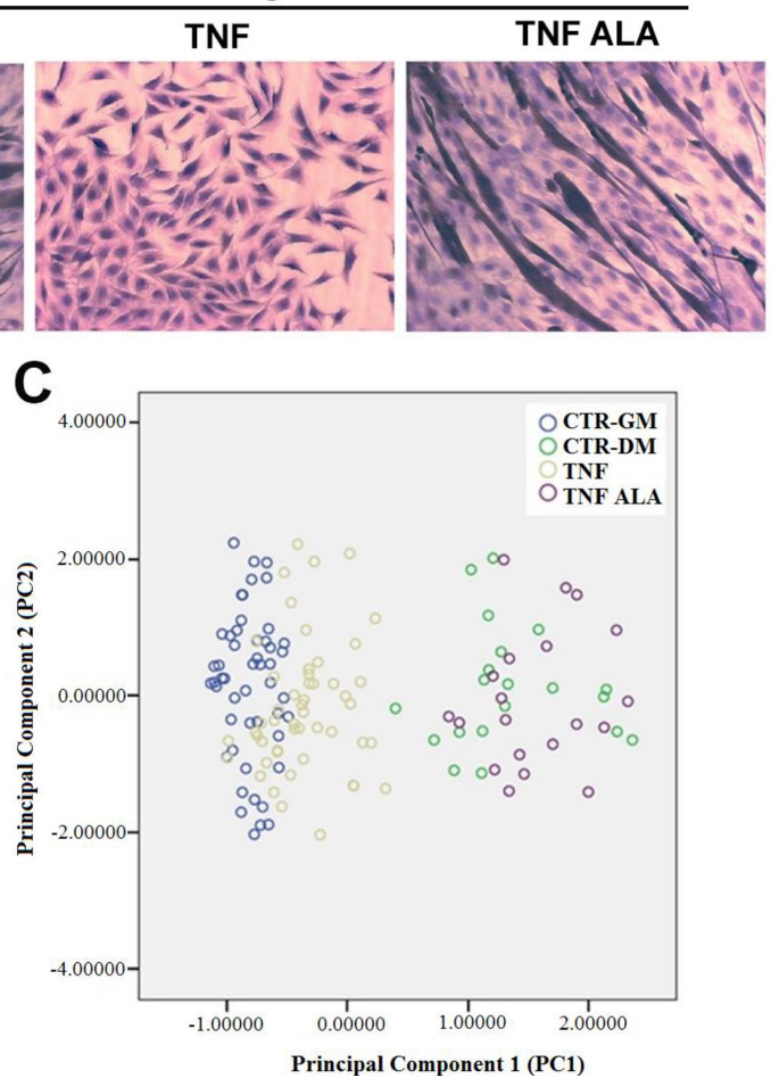

Figure 4. ALA preserves TNF-induced alterations on $\mathrm{C} 2 \mathrm{Cl} 2$ cell morphology during differentiation. Cells cultured in differentiating medium (DM) were untreated (CTR-DM) or treated with TNF in the absence (TNF) or presence of a-linolenic acid (TNF ALA) for 5 days. Cells cultured in growth medium (CTR-GM) served as negative controls. (A) Hematoxylin and eosin (H\&E) staining of C2C12 cells. Scale bar: $50 \mu \mathrm{m}$. (B) Component Score Coefficient Matrix. The coefficients by which variables are multiplied to obtain factor scores are shown. The variables are represented by the morphometric parameters derived from light microscope images of $\mathrm{C} 2 \mathrm{C} 12$ cells. The values highlighted (red boxes) indicate the variables mostly closely associated with Principal Components 1 and 2 . (C) Principal Component Analysis(PCA). The diagram shows the values from different experimental groups: CTR-GM, Blue dots; CTR-DM, green dots; TNF, yellow dots; TNF ALA, purple dots. The analysis indicates that the presence of ALA in the TNF-supplemented medium (TNF ALA) radically changed the morphological parameters of the C2C12 phenotype, restoring values that approximated those of differentiated cells (CTR-DM). 
The total variance explained by the PC1 and PC2 was $64.6 \%$, which may be considered an excellent result. As shown in Figure 4C, the PC1 varied markedly in untreated $\mathrm{C} 2 \mathrm{C} 12$ cells grown in differentiating medium (CTR-DM, green dots) when compared with cells grown in GM (CTR-GM, blue dots). TNF in the $\mathrm{DM}$ (TNF, yellow dots) reduced the PC1, restoring morphological parameter values that approximated those of undifferentiated cells (CTR-GM). The addition of ALA in the medium (TNF ALA, purple dots) increased the PC1 and completely changed the morphological parameter values of the $\mathrm{C} 2 \mathrm{C} 12$ phenotype, restoring the Area, Perim, AR, Num Nuclei, Circ, Round and Solidity parameter values (associated with PC1) that approximated those of the CTR-DM features. These results suggest that PCA analysis used for the quantification of morphological abnormalities in cellular and tissue of muscle disease models may be an excellent early predictor of such diseases.

\section{ALA reduces TNF-induced cell death and inhibition of differentiation on $\mathrm{C} 2 \mathrm{C} 12$ cells}

To further investigate whether the ALA effects on myogenesis may be due to protection against myoblasts death, TUNEL analysis was carried out (Fig. 5A). ALA reduced significantly the TNF-induced apoptosis in $\mathrm{C} 2 \mathrm{C} 12$ cells, after 2 days in DM, as evidenced from the quantitative data reported in Fig. 5B. To test expression of differentiation markers, immunofluorescence analysis was performed (Fig. 6). As expected, TNF inhibited C2C12 differentiation, after 5 days in DM, decreasing the number of positive myogenin nuclei and positive Myosin Heavy Chain (MHC) and caveolin-3 cells in respect to untreated cells (Fig. 6, A, C, F). ALA addition to DM restored the expression of myogenin, MHC and caveolin-3 in C2C12 cells after 5 days of treatment. (Fig. 6, A,C,F). In addition, ALA induced myoblast fusion that was significantly inhibited by TNF (Fig. 6 E). Quantitative data are reported in Fig. 6B, D, E and G.

\section{In Silico}

\section{Predicted pathways affected by n-3 PUFAs}

One explanation for the experimental in vivo and in vitro results was hypothesized to be the involvement of microRNAs. The following murine miRNAs associated with muscle differentiation were selected following a search in the literature: miR-23a, miR-24, miR-26a, miR-29a, miR-181a, miR-214 and miR-378, miR-1, miR-133 and miR-206 [41-52]. The search revealed that four of the aforementioned murine microRNAs, i.e miR-206, miR-181a, miR-29a and miR-26a, involved in murine $\mathrm{C} 2 \mathrm{C} 12$ muscle differentiation are also modulated by n-3 PUFAs in human cells $[25,53]$. These murine microRNAs, which are equivalent to human microRNAs, were therefore considered in the present research. In addition, the search in the literature highlighted that a further four miRNAs, i.e. miR-1, miR-133a, miR-133b and miR-206, involved in $\mathrm{C} 2 \mathrm{C} 12$ cell differentiation are modulated by TNF in the same cells [54]. It is noteworthy that miR-206 is modulated by both n-3 PUFAs and TNF. Therefore, the search in the literature yielded the following seven miRNAs for the computational analysis: miR-206, miR-181a, miR-29a, miR-26a, miR-1, miR-133a and miR-133b. The web-based computational database DIANA-MicroT 3.0 was used to extract the KEGG pathway IDs potentially altered by the expression of these seven miRNAs. These pathways were then filtered using the SID1.0 program to detect the common pathways. The database selected 149 common pathways ( $p$-value range between 0.05 and 32.36). Table 1 shows all the common pathways with $\mathrm{p}$ values above 10 (13 common pathways). The common pathways selected included those related to the cell-cell and cell-ECM interaction, such as the FAK pathway and pathways related to communication between the external microenvironment and the interior of the cells (DNA), such as MAPK.

\section{Discussion}

Dystrophy is a disease that is characterized by progressive muscle degeneration and weakness with cycles of muscle necrosis and regeneration as its pathophysiological hallmarks [4,5]. Although many therapeutic strategies have been proposed to counteract dystrophy, clinically effective treatments are not yet available [55].

Table 1. Predict Pathways involved in the beneficial effects of $n-3$ PUFAs on the impaired myogenesis

\begin{tabular}{lll}
\hline KEGG Pathway & Pathway ID & $-\ln (\mathrm{p}$-value) \\
\hline Focal adhesion & mmu04510 & 32.36 \\
Adherens junction & mmu04520 & 15.1 \\
ECM-receptor interaction & mmu04512 & 14.96 \\
Axon guidance & mmu04360 & 14.43 \\
MAPK signaling pathway & mmu04010 & 13.9 \\
Glioma & mmu05214 & 13.78 \\
Regulation of actin cytoskeleton & mmu04810 & 13.23 \\
GnRH signaling pathway & mmu04912 & 12.65 \\
Chronic myeloid leukemia & mmu05220 & 12.49 \\
Renal cell carcinoma & mmu05211 & 11.09 \\
Long-term potentiation & mmu04720 & 11.09 \\
TGF-beta signaling pathway & mmu04350 & 10.1 \\
mTOR signaling pathway & mmu04150 & 10.08 \\
Common Pathways (Kegg pathway ID) of seven murine miRNAs involved in \\
murine skeletal muscle differentiation: miR-206 (n-3 PUFAs and TNF modulated), \\
miR-181a, miR-29a and miR-26a (n-3 PUFAs modulated) and miR-1, miR-133a and \\
miR-133b ( TNF modulated). &
\end{tabular}




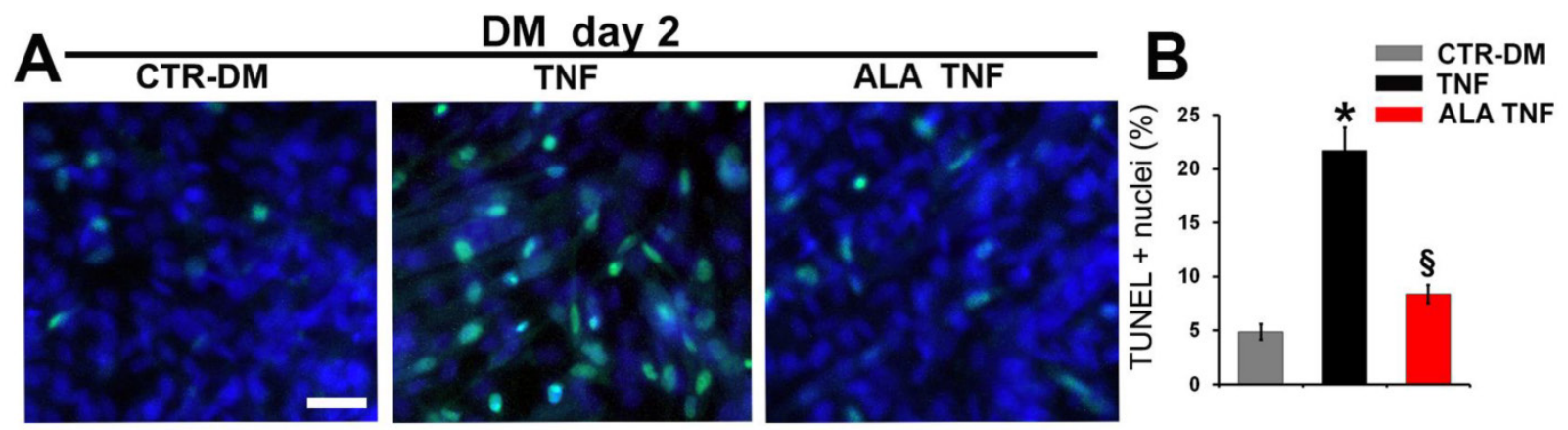

Figure 5. ALA precludes TNF-induced apoptosis in differentiating $\mathrm{C} 2 \mathrm{Cl} 2$ cells. Cells cultured in differentiating medium (DM) were untreated (CTR-DM) or treated with TNF in the absence (TNF) or presence of $\alpha$-linolenic acid (TNF ALA) for 2 days. (A) TUNEL analysis of $C 2 \mathrm{Cl} 2$ cells. Nuclei were stained with DAPI (blue) and apoptotic nuclei were decorated in green. Scale bar: $10 \mu \mathrm{m}$. (B) Quantitative analysis of TUNEL-positive nuclei presented as a percentage of total DAPI-stained nuclei. $* P<0.05$ vs. CTR; $\S P<0.05$ vs. TNF; $n$ (number of cell wells with the same treatment) $=5$

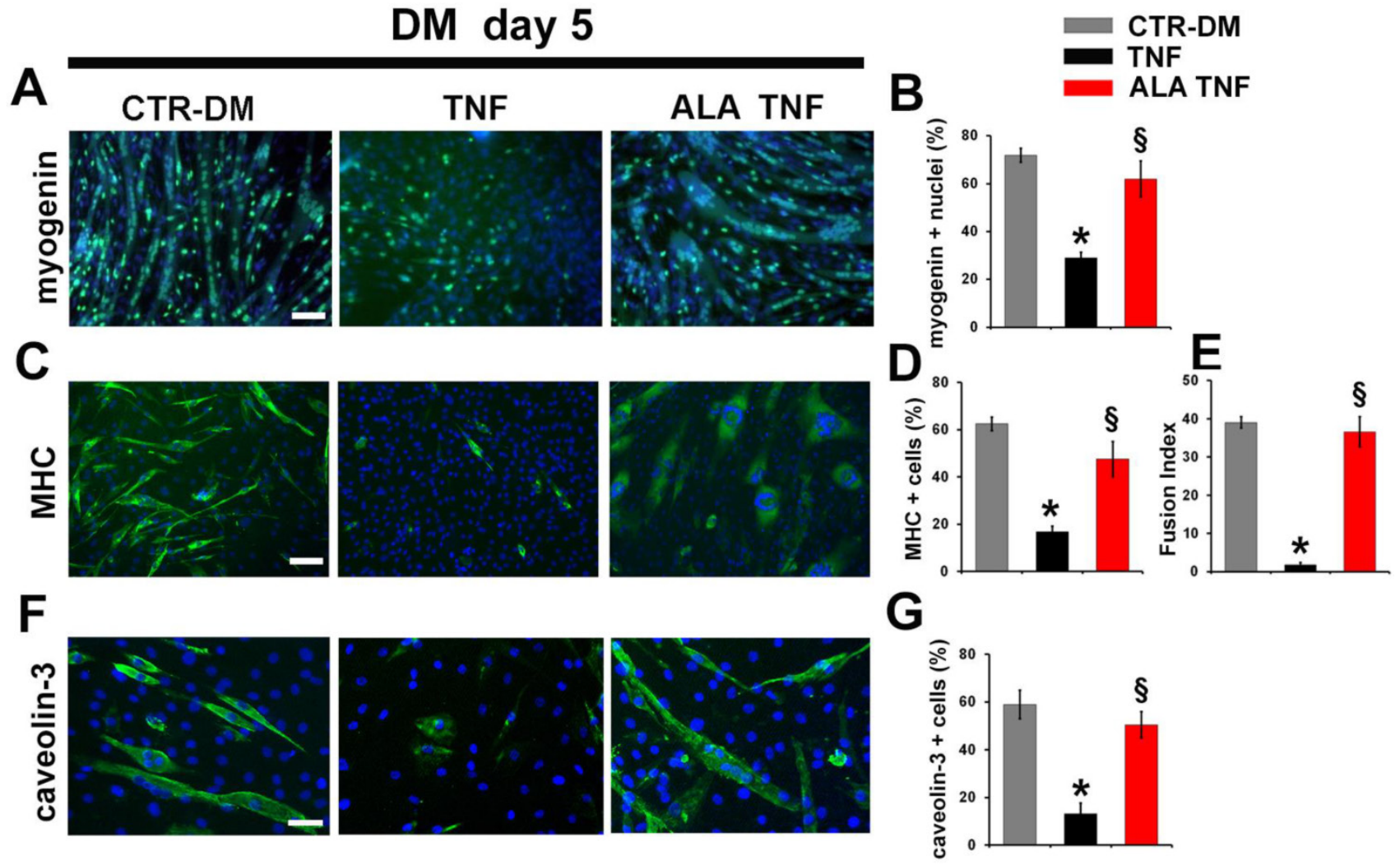

Figure 6. ALA induces muscle differentiation marker expression in differentiating C2C12 cells in the presence of TNF. Cells cultured in differentiating medium (DM) were untreated (CTR-DM) or treated with TNF in the absence (TNF) or presence of $\alpha$-linolenic acid (TNF ALA) for 5 days. (A) Immunofluorescence detection of myogenin (green) in C2C12 cells. Scale bar: $50 \mu \mathrm{m}$. (B) Percentage of myogenin positive nuclei out of all DAPI-stained nuclei. $* P<0.05$ vs. CTR-DM; $\$ P<0.05$ vs. TNF; $\mathrm{n}=5$. (C) Immunofluorescence detection of MHC (green) in C2C12 cells. Scale bar: $50 \mu \mathrm{m}$. (D) Percentage of MHC positive C2C12 cells out of all DAPI-stained nuclei. $* P<0.05$ vs. $C T R-D M ; ~ \$ P<0.001$ vs. TNF; $n=5$. (E) Fusion Index calculated as the average number of nuclei in $M H C$ positive $C 2 C 12$ cells containing at least three nuclei above the total number of nuclei. $* P<0.05$ vs. CTR-DM; $§ P<0.05$ vs. TNF; $n=5$. (F) Immunofluorescence detection of caveolin-3 (green) in $C 2 C 12$ cells. Scale bar: $30 \mu \mathrm{m}$. (G) Percentage of caveolin-3 positive cells out of all DAPI-stained nuclei. $* P<0.05$ vs. CTR-DM; $\S P<0.05$ vs. TNF; $n=5$. In $\mathbf{A}$, $\mathbf{C}$ and $\mathbf{F}$ nuclei were stained with DAPI (blue).

The present investigation reveals that flaxseed exerts a significant protective effect against muscle damage induced by impaired tissue regeneration. Dystrophic hamsters, used in the present study as an in vivo model, displayed extensive muscle degeneration at 150 days of age, when high levels of TNF and extensive tissue inflammation were reported $[12,13]$.

The FS diet mitigated the histological features of dystrophic skeletal muscles by preserving the differentiated morphology of the myofibers. This finding was confirmed by the PCA analysis, a statistical tool used to predict the effectiveness of treatments, in both in vivo and in vitro experiments, by a direct and objective visualization of the morphological changes in a two-dimensional plot. Besides preserving the muscle tissue architecture, the FS diet restores the biochemi- 
cal expression pattern of myogenesis in dystrophic muscle. At least two mechanisms may account for the protective effects displayed by the FS diet on myogenesis: (i) an anti-apoptotic action on differentiating skeletal muscle cells and (ii) the regulation of the expression of key proteins involved in differentiation. Our results show that, in dystrophic muscles, the FS diet reduces the number of apoptotic nuclei, particularly in interstitial cells, thus pointing to a possible protective effect on resident stem cells. Previous studies have reported that, although necrosis is probably the leading cause of myofiber degradation in many muscular dystrophies, apoptosis plays a paramount role in the depletion of satellite cells [56, 57]. Thus, the suppression of myoblast apoptosis may be an important mechanism underlying the protective effects of FS on dystrophic muscles. In addition, the FS diet reduces the number of Pax7 positive cells in the dystrophic skeletal muscles while enhancing the number of cells expressing myogenesis markers, such as myogenin, $\alpha-\mathrm{MHC}$ and caveolin-3. The FS diet thus appears to lead stem cell progression towards a differentiating stage. The beneficial effects of the FS diet on dystrophic muscles are further supported by the observation that FS not only increases the quantity of caveolin-3, but also allows its retention at the sarcolemma. Caveolin-3, which is prerequisite for caveolae formation in striated muscles [58], plays a pivotal role in cardiac [59] and skeletal [22] muscle protection as well as in sarcolemma membrane repair [60]. In addition, it favors cell muscle differentiation through myoblast fusion and myotube formation [23]. Lastly, caveolin-3 binds to beta dystroglycan, thereby contributing to the dystrophin-glycoprotein complex, which in turn links the cytoskeleton to the extracellular matrix [61]. The absence of the $\delta$-sarcoglycan in dystrophic hamster muscles impairs the whole dystrophin-glycoprotein complex, which is indispensable to membrane integrity during muscle contraction, calcium homeostasis and survival signaling [7, 62]. Our previous studies have shown that the FS diet partly restores the expression pattern of the dystrophin-glycoprotein complex membrane proteins in dystrophic skeletal muscles [12]. Therefore, restoration of the aberrant expression of caveolin-3 induced by the FS diet exerts a beneficial effect on the overall expression pattern of membrane signaling proteins in dystrophic muscles.

These beneficial effects of FS diet may be due, at least in part, to the modulation of the inflammatory microenvironment of the dystrophic muscle, as demonstrated by the decreased number of CD45 cells. In keeping with this hypothesis, previous studies have reported an anti-inflammatory action of dietary flaxseed at the cellular and molecular levels that was attributed mainly to the ALA content of flaxseed [9, 10]. It is noteworthy that plasma ALA levels increase significantly after ingestion of flaxseed in both humans [10, 63] and animals [9], including dystrophic hamsters [13]. ALA levels in the muscular tissue are also high in dystrophic hamsters on a flaxseed diet $[12,13]$.

TNF plasma levels were not, however, significantly lowered by the FS diet in our study. Since TNF may induce a bimodal myogenic response in muscle cells depending on its concentration, ALA may be hypothesized to change TNF signaling. Indeed, in previous studies, pathological levels of TNF have been associated with skeletal muscle apoptosis and inhibition of differentiation [16, 17, 64], while cytokine physiological concentrations have been reported to activate myogenesis [65].

The in vitro experiments we performed unequivocally revealed a direct effect of ALA on differentiating myoblasts. ALA reduced apoptosis in differentiating myoblasts even in the presence of high concentrations of TNF. In addition, ALA prevented the TNF-induced inhibition of myogenesis, as demonstrated by the increased expression of myogenin, MHC and caveolin-3 in C2C12 cells, while promoting myotube fusion. Therefore, ALA may modulate the dichotomy of TNF signalling properties that favor myogenesis and the survival signal pathways as opposed to the apoptotic pathways.

Taken together, our in vivo and in vitro results show that, besides reducing fibrosis and improving muscle structure of dystrophic hamsters [12], the FS diet enhances the survival of myoblasts and preserves their differentiation, probably owing to an ALA-mediated action. The FS diet consequently reverts the myocyte degeneration that is typical of dystrophic muscles. The upstream mechanisms of these effects may be due to: (i) the incorporation of ALA into the membrane and the subsequent alteration of the location of the TNF receptor domains in the caveolae/non-caveolae lipid regions of muscle cell membranes or (ii) an epigenetic effect on the transcription of genes for proteins involved in cell muscle protection and differentiation, such as caveolin-3. Since n-3 PUFAs may act through epigenetic mechanisms [25, 66] and miRNAs play an important role in myogenesis [28], it may be speculated that miRNA expression is modulated by $\mathrm{n} 3$ fatty acid ALA. An in silico analysis on a total of seven microRNAs, selected from a search in the literature, uncovered the possible altered signal pathways involved in the effects exerted by $n-3$ PUFAs on myogenesis.

The in silico analysis suggests that the focal adhesion kinase (FAK) pathway that exhibits the highest $p$ value (32.36) may play a central role in the regula- 
tion of myogenesis by ALA. The FAK pathway regulates cellular processes such as migration, growth, survival and differentiation [67]. In skeletal muscle, FAK signaling has been shown to regulate the expression of caveolin- 3 and $\beta 1$ integrin, two genes that are essential for myoblast fusion [68]. Therefore, ALA may indirectly regulate caveolin-3 expression through the FAK pathway. Since FAK is at the intersection of many signaling pathways, it is likely to regulate myogenesis by targeting multiple pathways. Pathways that are linked to communications between the external microenvironment and the interior of the cells (DNA), such as MAPK, may also be involved. Lastly, pathways, such as mTOR, which are involved in the balance between hypertrophy-atrophy/survivaldeath in skeletal muscle and mediate cellular responses to nutrients [69], may play an important role in orchestrating the beneficial effects of ALA on skeletal muscle. Indeed, the activation of both the p38 MAPK and PI3-kinase/Akt/mTOR pathways is required for proper caveolin-3 up-regulation during muscle differentiation [70]. Although each in silico prediction will need to be validated experimentally, these results provide a good starting point for future investigations into the role of n-3 PUFAs in the regulation of myogenesis.

In conclusion, the present study demonstrates that the pathological status of muscular dystrophy can be effectively mitigated by administering natural compounds contained in flaxseed that have a pleiotropic effect rather than by targeting a specific gene mutation as occurs in other therapeutic strategies [71]. Although the beneficial effects appear to be due above all to the ALA component of flaxseed, as is suggested by vitro experiments, the possible involvement of other flaxseed components that induce or enhance those effects, such as lignans and fibers, cannot be ruled out and warrant further investigation. Taken together, our findings suggest that flaxseed not only repairs the damage in the sarcolemma, which is the primary consequence of gene mutation, but also exerts a more extensive epigenetic action that stimulates multiple molecular pathways in dystrophic muscles.

The prolonged exposure of dystrophic muscles (from weaning to death) to flaxseed components such as ALA would presumably allow a constant modulation of the muscle microenvironment that would, in turn, effectively alleviate muscle damage. Thus, administration of flaxseed and ALA to patients with muscular dystrophies may be beneficial in the clinical setting

\section{Abbreviations}

n-3 PUFAs: n-3 polyunsaturated fatty acids; TNF: tumor necrosis factor- $\alpha$; ALA: $\alpha$-linolenic acid;
FS: flaxseed-enriched diet; miRNAs: microRNAs; Dystr/P: dystrophic hamster fed with standard pellet; Dystr/FS: dystrophic hamster fed with flaxseed; PCA: Principal Component Analysis; MHC: Myosin Heavy Chain; TRITC-WGA: TRITC-Wheat germ agglutinin; FAK: focal adhesion kinase.

\section{Acknowledgments}

This work was funded by Fondazione San Raffaele, Ceglie Messapica Italy Research Project MERIT-RBNE08HM7T - Italian Ministry of Health and partially by ANR (no. 13-BSV1-0005), NIH (no. 5R01CA180057-02), AFM (no. 2012-0773), UPMC Emergence 2011, and IBPS 2015.

The authors would like to thank Dott. Mauro Sansò, Chief Operating Officer at Fondazione San Raffaele for his excellent management of the MERIT \#RBNE08HM7T funds.

\section{Competing interests}

The authors declare that they have no competing interests

\section{References}

1. Yin H, Price F, Rudnicki MA. Satellite cells and the muscle stem cell niche. Physiol Rev. 2013; 93: 23-67.

2. Mauro A. Satellite cell of skeletal muscle fibers. J Biophys Biochem Cytol. 1961; 9: 493-5

3. Hawke TJ, Garry DJ. Myogenic satellite cells: physiology to molecular biology. J Appl Physiol (1985). 2001; 91: 534-51.

4. Musarò A. The Basis of Muscle Regeneration. Advances in Biology; 2014. p. 16.

5. Wallace GQ, McNally EM. Mechanisms of muscle degeneration, regeneration, and repair in the muscular dystrophies. Annu Rev Physiol. 2009; 71: 37-57.

6. Emery AE. The muscular dystrophies. Lancet. 2002; 359: 687-95.

7. Gumerson JD, Michele DE. The dystrophin-glycoprotein complex in the prevention of muscle damage. J Biomed Biotechnol. 2011; 2011: 210797.

8. Vel Szic KS, Declerck K, Vidakovic M, Vanden Berghe W. From inflammaging to healthy aging by dietary lifestyle choices: is epigenetics the key to personalized nutrition? Clin Epigenetics. 2015; 7: 33.

9. Dupasquier CM, Dibrov E, Kneesh AL, Cheung PK, Lee KG, Alexander HK, et al. Dietary flaxseed inhibits atherosclerosis in the LDL receptor-deficient mouse in part through antiproliferative and anti-inflammatory actions. Am J Physiol Heart Circ Physiol. 2007; 293: H2394-402.

10. Caligiuri SP, Aukema HM, Ravandi A, Guzman R, Dibrov E, Pierce GN Flaxseed consumption reduces blood pressure in patients with hypertension by altering circulating oxylipins via an alpha-linolenic acid-induced inhibition of soluble epoxide hydrolase. Hypertension. 2014; 64: 53-9.

11. Rodriguez-Leyva D, Dupasquier CM, McCullough R, Pierce GN. The cardiovascular effects of flaxseed and its omega-3 fatty acid, alpha-linolenic acid. Can J Cardiol. 2010; 26: 489-96.

12. Fiaccavento R, Carotenuto F, Vecchini A, Binaglia L, Forte G, Capucci E, et al. An omega-3 fatty acid-enriched diet prevents skeletal muscle lesions in a hamster model of dystrophy. Am J Pathol. 2010; 177: 2176-84.

13. Carotenuto F, Minieri M, Monego G, Fiaccavento R, Bertoni A, Sinigaglia F, et al. A diet supplemented with ALA-rich flaxseed prevents cardiomyocyte apoptosis by regulating caveolin-3 expression. Cardiovasc Res. 2013; 100: 422-31.

14. Fiaccavento R, Carotenuto F, Minieri M, Masuelli L, Vecchini A, Bei R, et al. Alpha-linolenic acid-enriched diet prevents myocardial damage and expands longevity in cardiomyopathic hamsters. Am J Pathol. 2006; 169: 1913-24.

15. Grounds MD, Radley HG, Gebski BL, Bogoyevitch MA, Shavlakadze T. Implications of cross-talk between tumour necrosis factor and insulin-like growth factor-1 signalling in skeletal muscle. Clin Exp Pharmacol Physiol. 2008; 35: 846-51.

16. Meadows KA, Holly JM, Stewart CE. Tumor necrosis factor-alpha-induced apoptosis is associated with suppression of insulin-like growth factor binding protein-5 secretion in differentiating murine skeletal myoblasts. J Cell Physiol. 2000; 183: 330-7.

17. Stewart CE, Newcomb PV, Holly JM. Multifaceted roles of TNF-alpha in myoblast destruction: a multitude of signal transduction pathways. J Cell Physiol. 2004; 198: 237-47. 
18. Coletti D, Moresi V, Adamo $\mathrm{S}$, Molinaro M, Sassoon D. Tumor necrosis factor-alpha gene transfer induces cachexia and inhibits muscle regeneration. Genesis. 2005; 43: 120-8.

19. Langen RC, Schols AM, Kelders MC, Wouters EF, Janssen-Heininger YM. Inflammatory cytokines inhibit myogenic differentiation through activation of nuclear factor-kappaB. FASEB J. 2001; 15: 1169-80.

20. Sellers SL, Trane AE, Bernatchez PN. Caveolin as a potential drug target for cardiovascular protection. Front Physiol. 2012; 3: 280.

21. Tsutsumi YM, Tsutsumi R, Horikawa YT, Sakai Y, Hamaguchi E, Ishikawa Y, et al. Geranylgeranylacetone protects the heart via caveolae and caveolin-3. Life Sci. 2014; 101: 43-8.

22. Tan Z, Zhou LJ, Mu PW, Liu SP, Chen SJ, Fu XD, et al. Caveolin-3 is involved in the protection of resveratrol against high-fat-diet-induced insulin resistance by promoting GLUT4 translocation to the plasma membrane in skeletal muscle of ovariectomized rats. J Nutr Biochem. 2012; 23: 1716-24.

23. Galbiati F, Volonte D, Engelman JA, Scherer PE, Lisanti MP. Targeted down-regulation of caveolin-3 is sufficient to inhibit myotube formation in differentiating $\mathrm{C} 2 \mathrm{C} 12$ myoblasts. Transient activation of p38 mitogen-activated protein kinase is required for induction of caveolin-3 expression and subsequent myotube formation. J Biol Chem. 1999; 274: 30315-21.

24. Ceccarelli V, Racanicchi S, Martelli MP, Nocentini G, Fettucciari K, Riccardi C, et al. Eicosapentaenoic acid demethylates a single $\mathrm{CpG}$ that mediates expression of tumor suppressor CCAAT/enhancer-binding protein delta in U937 leukemia cells. J Biol Chem. 2011; 286: 27092-102.

25. Visioli F, Giordano E, Nicod NM, Davalos A. Molecular targets of omega 3 and conjugated linoleic Fatty acids - "micromanaging" cellular response. Front Physiol. 2012; 3: 42.

26. Lee HS, Barraza-Villarreal A, Hernandez-Vargas H, Sly PD, Biessy C, Ramakrishnan U, et al. Modulation of DNA methylation states and infant immune system by dietary supplementation with omega-3 PUFA during pregnancy in an intervention study. Am J Clin Nutr. 2013; 98: 480-7.

27. Yan X, Chao T, Tu K, Zhang Y, Xie L, Gong Y, et al. Improving the prediction of human microRNA target genes by using ensemble algorithm. FEBS Lett. 2007; 581: 1587-93.

28. Luo W, Nie Q, Zhang X. MicroRNAs involved in skeletal muscle differentiation. J Genet Genomics. 2013; 40: 107-16.

29. Lafferty-Whyte K, Cairney CJ, Jamieson NB, Oien KA, Keith WN. Pathway analysis of senescence-associated miRNA targets reveals common processes to different senescence induction mechanisms. Biochim Biophys Acta. 2009; 1792: 341-52.

30. Albertini MC, Olivieri F, Lazzarini R, Pilolli F, Galli F, Spada G, et al. Predicting microRNA modulation in human prostate cancer using a simple String IDentifier (SID1.0). J Biomed Inform. 2011; 44: 615-20.

31. Rippo MR, Olivieri F, Monsurro V, Prattichizzo F, Albertini MC, Procopio AD. MitomiRs in human inflamm-aging: a hypothesis involving miR-181a, miR-34a and miR-146a. Exp Gerontol. 2014; 56: 154-63.

32. Blain AM, Straub VW. delta-Sarcoglycan-deficient muscular dystrophy: from discovery to therapeutic approaches. Skelet Muscle. 2011; 1: 13.

33. Gebauer SK, Psota TL, Harris WS, Kris-Etherton PM. n-3 fatty acid dietary recommendations and food sources to achieve essentiality and cardiovascular benefits. Am J Clin Nutr. 2006; 83: 1526S-35S.

34. Albertini MC, Accorsi A, Teodori L, Pierfelici L, Uguccioni F, Rocchi MB, et al Use of multiparameter analysis for Vibrio alginolyticus viable but nonculturable state determination. Cytometry A. 2006; 69: 260-5.

35. Gonzalez RC, Woods RE. Digital Image Processing. Engewood Cliffs, NJ Prentice Hall; 2002.

36. Barret H. Foundations of Image Science. third ed. New Jersey, UK: John Wiley \& Sons; 2004.

37. Anderson TW. An Introduction to Multivariate Statistical Analysis. New York: Wiley; 1984

38. Burgess A, Vigneron S, Brioudes E, Labbe JC, Lorca T, Castro A. Loss of human Greatwall results in G2 arrest and multiple mitotic defects due to deregulation of the cyclin B-Cdc2/PP2A balance. Proc Natl Acad Sci U S A. 2010; 107: $12564-9$

39. Papadopoulos GL, Alexiou P, Maragkakis M, Reczko M, Hatzigeorgiou AG. DIANA-mirPath: Integrating human and mouse microRNAs in pathways. Bioinformatics. 2009; 25: 1991-3.

40. Szalay K, Razga Z, Duda E. TNF inhibits myogenesis and downregulates the expression of myogenic regulatory factors myoD and myogenin. Eur J Cell Biol. 1997; 74: 391-8.

41. Wang L, Chen X, Zheng Y, Li F, Lu Z, Chen C, et al. MiR-23a inhibits myogenic differentiation through down regulation of fast myosin heavy chain isoforms. Exp Cell Res. 2012; 318: 2324-34.

42. Sun Q, Zhang Y, Yang G, Chen X, Zhang Y, Cao G, et al. Transforming growth factor-beta-regulated miR-24 promotes skeletal muscle differentiation. Nucleic Acids Res. 2008; 36: 2690-9.

43. Kang WJ, Cho YL, Chae JR, Lee JD, Choi KJ, Kim S. Molecular beacon-based bioimaging of multiple microRNAs during myogenesis. Biomaterials. 2011;32: 1915-22.

44. Wang XH, Hu Z, Klein JD, Zhang L, Fang F, Mitch WE. Decreased miR-29 suppresses myogenesis in CKD. J Am Soc Nephrol. 2011; 22: 2068-76.

45. Winbanks CE, Wang B, Beyer C, Koh P, White L, Kantharidis P, et al. TGF-beta regulates miR-206 and miR-29 to control myogenic differentiation through regulation of HDAC4. J Biol Chem. 2011; 286: 13805-14
46. Zhou L, Wang L, Lu L, Jiang P, Sun H, Wang H. Inhibition of miR-29 by TGF-beta-Smad 3 signaling through dual mechanisms promotes transdifferentiation of mouse myoblasts into myofibroblasts. PLoS One. 2012; 7: e33766.

47. Bhushan R, Grunhagen J, Becker J, Robinson PN, Ott CE, Knaus P. miR-181a promotes osteoblastic differentiation through repression of TGF-beta signaling molecules. Int J Biochem Cell Biol. 2013; 45: 696-705.

48. Feng Y, Cao JH, Li XY, Zhao SH. Inhibition of miR-214 expression represses proliferation and differentiation of $\mathrm{C} 2 \mathrm{C} 12$ myoblasts. Cell Biochem Funct. 2011; 29: 378-83

49. Liu J, Luo XJ, Xiong AW, Zhang ZD, Yue S, Zhu MS, et al. MicroRNA-214 promotes myogenic differentiation by facilitating exit from mitosis via down-regulation of proto-oncogene N-ras. J Biol Chem. 2010; 285: 26599-607.

50. Gagan J, Dey BK, Layer R, Yan Z, Dutta A. MicroRNA-378 targets the myogenic repressor MyoR during myoblast differentiation. J Biol Chem. 2011; 286: 19431-8

51. Goljanek-Whysall K, Pais H, Rathjen T, Sweetman D, Dalmay T, Munsterberg A. Regulation of multiple target genes by miR-1 and miR-206 is pivotal for 2C12 myoblast differentiation. J Cell Sci. 2012; 125: 3590-600.

52. Sweetman D, Goljanek K, Rathjen T, Oustanina S, Braun T, Dalmay T, et al. Specific requirements of MRFs for the expression of muscle specific microRNAs, miR-1, miR-206 and miR-133. Dev Biol. 2008; 321: 491-9.

53. Farago N, Feher LZ, Kitajka K, Das UN, Puskas LG. MicroRNA profile of polyunsaturated fatty acid treated glioma cells reveal apoptosis-specific expression changes. Lipids Health Dis. 2011; 10: 173.

54. Georgantas RW, Streicher K, Greenberg SA, Greenlees LM, Zhu W, Brohawn PZ, et al. Inhibition of myogenic microRNAs 1, 133, and 206 by inflammatory cytokines links inflammation and muscle degeneration in adult inflammatory myopathies. Arthritis Rheumatol. 2014; 66: 1022-33.

55. Berardi E, Annibali D, Cassano M, Crippa S, Sampaolesi M. Molecular and cell-based therapies for muscle degenerations: a road under construction. Front Physiol. 2014; 5: 119

56. Sandri M, Minetti C, Pedemonte M, Carraro U. Apoptotic myonuclei in human Duchenne muscular dystrophy. Lab Invest. 1998; 78: 1005-16.

57. Hack AA, Ly CT, Jiang F, Clendenin CI, Sigrist KS, Wollmann RL, et al. Gamma-sarcoglycan deficiency leads to muscle membrane defects and apoptosis independent of dystrophin. J Cell Biol. 1998; 142: 1279-87.

58. Parton RG, del Pozo MA. Caveolae as plasma membrane sensors, protectors and organizers. Nat Rev Mol Cell Biol. 2013; 14: 98-112.

59. Tsutsumi YM, Horikawa YT, Jennings MM, Kidd MW, Niesman IR, Yokoyama U, et al. Cardiac-specific overexpression of caveolin-3 induces endogenous cardiac protection by mimicking ischemic preconditioning. Circulation. 2008; 118: 1979-88.

60. He B, Tang RH, Weisleder N, Xiao B, Yuan Z, Cai C, et al. Enhancing muscle membrane repair by gene delivery of MG53 ameliorates muscular dystrophy and heart failure in delta-Sarcoglycan-deficient hamsters. Mol Ther. 2012; 20: 727-35.

61. Sotgia F, Lee JK, Das K, Bedford M, Petrucci TC, Macioce P, et al. Caveolin-3 directly interacts with the C-terminal tail of beta -dystroglycan. Identification of a central WW-like domain within caveolin family members. J Biol Chem. 2000; 275: 38048-58.

62. Straub V, Duclos F, Venzke DP, Lee JC, Cutshall S, Leveille CJ, et al. Molecular pathogenesis of muscle degeneration in the delta-sarcoglycan-deficient hamster. Am J Pathol. 1998; 153: 1623-30.

63. Austria JA, Richard MN, Chahine MN, Edel AL, Malcolmson LJ, Dupasquier $\mathrm{CM}$, et al. Bioavailability of alpha-linolenic acid in subjects after ingestion of three different forms of flaxseed. J Am Coll Nutr. 2008; 27: 214-21.

64. Tolosa L, Morla M, Iglesias A, Busquets X, Llado J, Olmos G. IFN-gamma prevents TNF-alpha-induced apoptosis in $\mathrm{C} 2 \mathrm{C} 12$ myotubes through down-regulation of TNF-R2 and increased NF-kappaB activity. Cell Signal. 2005; 17: 1333-42.

65. Chen SE, Jin B, Li YP. TNF-alpha regulates myogenesis and muscle regeneration by activating p38 MAPK. Am J Physiol Cell Physiol. 2007; 292: C1660-71.

66. Tsoukas MA, Ko BJ, Witte TR, Dincer F, Hardman WE, Mantzoros CS. Dietary walnut suppression of colorectal cancer in mice: Mediation by miRNA patterns and fatty acid incorporation. J Nutr Biochem. 2015.

67. Mitra SK, Hanson DA, Schlaepfer DD. Focal adhesion kinase: in command and control of cell motility. Nat Rev Mol Cell Biol. 2005; 6: 56-68.

68. Quach NL, Biressi S, Reichardt LF, Keller C, Rando TA. Focal adhesion kinase signaling regulates the expression of caveolin 3 and beta1 integrin, genes essential for normal myoblast fusion. Mol Biol Cell. 2009; 20: 3422-35.

69. De Palma C, Perrotta C, Pellegrino P, Clementi E, Cervia D. Skeletal muscle homeostasis in duchenne muscular dystrophy: modulating autophagy as a promising therapeutic strategy. Front Aging Neurosci. 2014; 6: 188

70. Fanzani A, Musaro A, Stoppani E, Giuliani R, Colombo F, Preti A, et al. Hypertrophy and atrophy inversely regulate Caveolin-3 expression in myoblasts. Biochem Biophys Res Commun. 2007; 357: 314-8.

71. Fairclough RJ, Wood MJ, Davies KE. Therapy for Duchenne muscular dystrophy: renewed optimism from genetic approaches. Nat Rev Genet. 2013; 14: 373-8. 